\title{
Use of Telomere Length as a Biomarker for Aging and Age-Related Disease
}

\author{
Michael Fossel
}

Published online: 13 April 2012

(C) Springer Science+Business Media, LLC 2012

\begin{abstract}
Telomeres (measured as leukocyte telomere length) can be effective and useful biomarkers for general aging status, for age-related disease diagnosis, and for disease status, but they are less effective as predictive biomarkers for patient mortality. Recent work also suggests that we may be able to intervene in this biomarker by re-lengthening telomeres, and that this intervention may have significant clinical benefits. A great deal remains unknown regarding the limits of telomere lengths as clinical biomarkers and the limits of interventions aimed at re-lengthening human telomeres.
\end{abstract}

Keywords Telomeres $\cdot$ Aging $\cdot$ Telomerase $\cdot$ Age-related disease $\cdot$ Biomarkers

\section{Introduction}

Aging and age-related diseases are often difficult to assess clinically, particularly in giving an accurate prognosis and estimate of mortality or in choosing an optimal intervention, prompting the use of biomarkers. In assessing a particular aging patient, the earliest and most common marker has simply been the patient's chronologic age. It is readily available, noninvasive, numeric, and generally both reliable and valid; we assess aging by the number of years since the patient's birth.

That chronologic age is simply a biomarker, and not the actual clinical result itself, is evident in the clinical truism that the rate of aging varies between patients and that the rate of aging varies within patients when comparing different organs

M. Fossel $(\bowtie)$

9464 Conservation Street Northeast,

Ada, MI 49301, USA

e-mail: michael.fossel@gmail.com or body systems within a single patient. We attribute the disparity between chronologic age and clinical age to genetic proclivities, microbial (and other) disease history, nutrition, environmental exposures, and a host of other individualizing factors.

The result is that not only do patients age at different rates, but within individual patients, tissues and organs likewise vary. One patient will lack cardiovascular pathology, yet have severe impairment due to osteoarthritis; another will have remarkably young-appearing skin, yet suffer from advanced Alzheimer's dementia. Patients and their systems vary. Though still useful, chronological age is an inaccurate biomarker, prompting the search for biomarkers with greater clinical utility and specificity, the better to assess current disease status, predict outcomes, and guide effective interventions.

As a result, clinicians currently use a plethora of (variably effective) biomarkers (eg, age, serum metabolite levels, physiological measures, and functional tests) to clarify and define the aging characteristics of patients. The more apt the biomarker we choose, the better is our ability to intervene in age-related clinical diseases.

\section{Defining Useful Biomarkers}

While any number of biomarkers can (and have been) defined, appropriate biomarkers share several practical characteristics:

First, a good biomarker must be reliably and sufficiently correlated with the underlying disease process. Cholesterol levels, for example, are generally (but not uniformly) correlated with atherosclerotic disease and with the risk of death due to that disease. There are clinical examples of patients with low cholesterol levels and high risk of mortality due to coronary artery disease (eg, Hutchinson-Gilford Progeria) 
[1] and of patients with high cholesterol who outlive their physicians. Equally to the point, there has been at least one example of a commercial drug that lowered serum cholesterol, yet raised mortality. Serum cholesterol is, in a general sense, a useful biomarker for mortality, and specifically coronary arterial disease, yet it has limits.

Second, a good biomarker must be clinically practical (ie, easy to obtain in both clinical and financial terms). One might argue that the best (ie, most sensitive and specific) biomarker for Alzheimer's dementia is based on a forebrain biopsy of the patient, but a neuropsychiatric evaluation, a family history, and identification of the apolipoprotein (apo) $\mathrm{E}$ alleles are much easier to obtain, and therefore constitute the more practical biomarkers. At the other extreme, chronologic age is easy to obtain, but has a lower predictive value than other biomarkers.

This, a good biomarker should vary with the particular disease (or tissue, organ, cell type) of interest, rather than reflect wholesale clinical status. A patient's chronological age may be good overall indicator of aging status, but it does not distinguish patients who are more likely to suffer from arterial aging versus those who are more likely to suffer from joint aging. In these diseases, we prefer a focal biomarker that indicates the specific status of the specific organ or tissue in question. A biomarker should tell us not only that our patient has a disease, but also which disease our patient has.

Finally, optimally, a biomarker will guide clinical intervention. If a biomarker sufficiently reflects the underlying process that it can serve as a surrogate point of intervention, then we want to be capable of providing such intervention. This is not always feasible; in the case of coronary artery disease, we know of at least three major variables that we can use as practical targets for clinical intervention (blood pressure, serum cholesterol, and smoking), but the most important biomarker (the risk factor of family history) is not amenable to clinical intervention. We prefer biomarkers that are not only accurate and easy to obtain, but are targets for clinical intervention. Family history is a good biomarker for predicting disease, but it lacks this final, highly desirable characteristic of permitting intervention. In this particular example, the underlying alleles would be a better biomarker (once practical genetic therapy is available) than the simplistic biomarker of family history.

\section{Telomeres in a Clinical Perspective}

Telomeres have been suggested as age-related biomarkers and as points for targeted clinical intervention for at least 15 years $[2,3]$, and the underlying genetic mechanisms and potential clinical utility have been reviewed elsewhere [4, 5]. Telomere lengths are determined by inheritance [6], but also affected by environmental factors [7]. There is a large and rapidly growing body of data showing that telomere lengths are directly related not only to cell aging, but to tissue and organ aging as well, and telomere length has been implicated in human age-related diseases, all of which was reviewed in extensive detail in a clinical textbook [8]. Despite the early clinical promise of telomeres as both diagnostic and therapeutic targets, initial reception was limited to basic research, with very little translational work into clinical medicine. Recently, this has begun to change, with a growing appreciation of the potential value for predicting clinical progression and disease severity, as well as translational research and intervention [9•].

In a more minatory vein, however, telomeres are once again the darling not only of the research community, but also of the public media as well, prompting considerable interest and many (usually unsubstantiated) public claims for clinical intervention. The medical community itself has shown a growing interest, limited by available clinical data and by commercially available techniques to assess telomere lengths. While several techniques have been available to measure telomere lengths within the research context, within the past year, two commercial enterprises, both founded by key telomere researchers, have begun to offer telomere length measurements to clinicians [10]. The first of these, Telome Health, was founded by Calvin Harley and Elizabeth Blackburn (www.telomehealth. com); the second, Life Length, was founded by Maria Blasco (www.lifelength.com). These enterprises are based on the belief that telomere lengths may serve as an appropriate biomarker for aging or for age-related diseases, and that this knowledge may have significant implications for clinical medicine.

\section{Measuring Telomere Lengths: Techniques}

To what extent are these claims justified in a clinical context? The answer to this question depends not only upon the implications of telomere lengths for aging and age-related disease, but also upon the reliability and validity of the techniques used to measure telomere lengths. Two techniques are available for clinical use at this time: quantitative FISH (fluorescence in situ hybridization) and polymerase chain reaction (PCR). Most current clinical data is based on the FISH technique, which measures the mean telomere lengths of a cell sample. Although having less available clinical data for comparison, the PCR technique offers both a mean and a value for the shortest telomeres in the sample. The FISH technique can be adapted to very small clinical samples, including saliva; both can be done on blood samples. Within those limitations, both techniques are probably both sufficiently reliable to permit effective clinical use, 
while the issue of validity (as an appropriate biomarker) will be discussed further later in this article.

Telomeres can be used as a biomarker, but is the measurement reliable? In the case of telomere lengths, unreliability can derive from several sources: inherent variation between samples of a single patient over time, variation between different techniques, and variation between different laboratories using the same technique. Several authors have noted that the measurements of telomere lengths in a single patient may vary over time, not only with the expected slow erosion of telomere base pairs with age, but also with an oscillation pattern or with other patterns that may be related to infection, environmental stress, and other factors. Svenson et al. [7], for example, found a 6-month periodic oscillation in one of their donors, and argue that their data "...support the concept that individual blood cell telomere length is a dynamic feature" with potential implications for future clinical use.

For over a decade, infectious disease has been known to cause changes in telomere lengths. While infectious disease in the long run tends to result in shorter leukocyte telomeres, in the short run an infection may serve to "recruit" leukocytes with longer telomeres, lending an inherent variance to such measurements and leading most researchers to stress the value of serial determinations of leukocyte telomere lengths rather crediting the reliability of any single measurement. For example, lymphocyte telomeres shorten in cytomegalovirus [11], hepatitis C [12], and HIV infections [13].

The most common sources of variance are probably the use of different techniques or of the same technique as performed in different laboratories [14]. To the extent that telomere lengths can be a reliable biomarker, a preference should be given to using a single technique and within a single laboratory, and an experienced laboratory rather than an inexperienced one. Whatever the laboratory or technique, many of the common methods of determining telomere lengths are "fraught with shortcomings that limit their use," although newer methods are proving simpler and more reliable, with lower variance, an ability to be precise despite degraded DNA, and adaptable to high throughput analysis [15].

\section{Telomeres Versus Other Current Biomarkers}

Clearly telomeres can be used as biomarkers, but is there anything to recommend them over other, more proven biomarkers? Here the question breaks down into two potential issues: the use of telomere lengths as a biomarker for aging per se, and the use of telomere lengths as a biomarker for individual diseases of aging. Although telomere length has long been felt to represent a more useful biomarker than is the chronologic age (because telomere lengths may more accurately reflect the environmental and genetic differences between individuals who display clinical aging at differing rates) and although this position has good theoretical and laboratory support [8], actual clinical support is somewhat more tenuous [16]. Part of this is attributable to our fuzzy concept of "aging" in opposition to the more utilitarian and well-defined concepts inherent in age-related diseases. A myocardial infarction is striking and welldefined, while the concept of whole-body aging remains vague in comparison.

Most authors, with a more practical, clinical bent, have looked at telomere lengths (or proteins associated with telomeres) [17] as biomarkers for diseases of aging rather than aging per se. Such diseases have run the gamut of human agerelated disease, but most work has concentrated on cardiovascular disease (especially coronary arterial disease), alterations in immune function, cancer, and (to a lesser extent) diseases of the central nervous system such as Alzheimer's dementia. Telomere length is associated with "age-related disease burden across multiple physiological systems," independent of chronologic age [18], but does this association carry over into individual diseases? Telomere shortening certainly varies among tissues and cells within tissues [17]. In the case of cardiovascular disease, for example, the cardiomyocytes (or in the case of Alzheimer's dementia, the neurons) show little change in telomere length, but the pathology is attributable to and correlated with the pathology of the endothelial cells of the coronary arteries (or in the case of Alzheimer's dementia, the microglial cells), which clearly do show shortening of their telomeres, and such shortening correlates with disease progression.

\section{Cardiovascular Disease}

Studies of cardiovascular disease have generally looked at the ability of telomere lengths to predict cardiovascular "events" (eg, myocardial infarction) or cardiovascular mortality (Figs. 1 and 2) [19, 20], and the conclusions have varied widely as to whether or not telomere lengths might serve as a clinically useful biomarker. Although on the whole, telomere lengths "can predict cardiovascular events," there have been problems with confounded variables, differences in technique, and different study populations [21], which have resulted in disparate opinions.

Telomere lengths, however, were not found to be predictive of mortality, specifically cardiovascular mortality, especially in men greater than age 70 years [22] or in those patients followed for 7 years [23], in whom body mass index (BMI) and smoking status were the more useful biomarkers. Short telomere lengths were only modestly useful in predicting myocardial infarction, ischemic disease, or death [24]. With regard to arterial pathology, leukocyte telomere lengths were good markers for the presence (but not the extent) of carotid plaques, as well as to re-stenosis 
MI Incidence

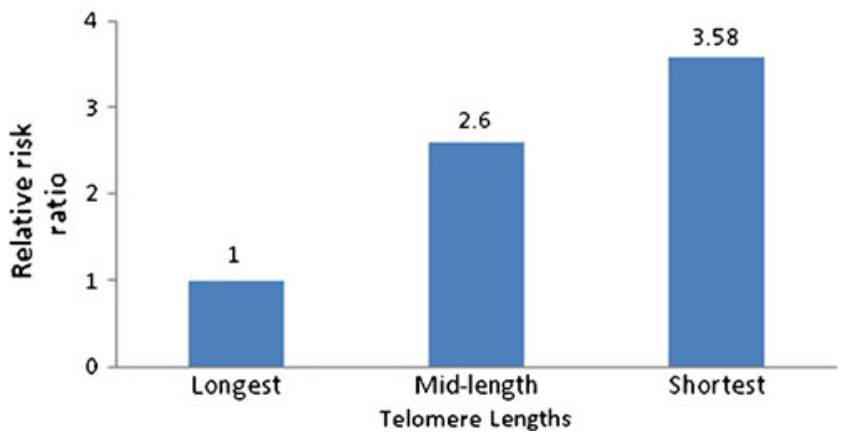

Fig. 1 Relation of telomere length to incidence of myocardial infarction (MI) (Data adapted from Willeit et al. [20])

[25]. Just as inheritance serves as a biomarker for many agerelated diseases, and specifically in the case of ischemic heart failure, telomeres are likewise useful biomarkers for this disease and, in fact, may be the heritable element (or one of the heritable elements) underlying the cascade of pathology that results in this familial pattern of disease [6].

Age-dependent telomere shortening occurs in most somatic cells that underlie age-related cardiovascular pathology (eg, vascular endothelial cells, smooth muscle cells, and leukocytes, among others), resulting in hypertension, atherosclerosis, and heart failure [26]. Similar relationships are found in iatrogenically injured tissues (eg, in balloon injury) and in cells undergoing oxidative stress in animal models [27]. While telomere length may not be associated with smoking status, BMI, blood pressure, or alcohol use, short telomere lengths (both mean length and proportion shorter than $5 \mathrm{~kb}$ ) are associated with coronary artery disease, transient ischemic attacks, and type 2 diabetes in many older patient populations [28]. Shortened telomere lengths, as measured in circulating leukocytes, are also associated with high-risk plaque morphology, perhaps as a result of increased inflammatory activity [29].

Telomeres appear to be questionable biomarkers for predicting cardiovascular mortality, but they show some promise as biomarkers of cardiovascular disease in general and perhaps cardiovascular events as well.

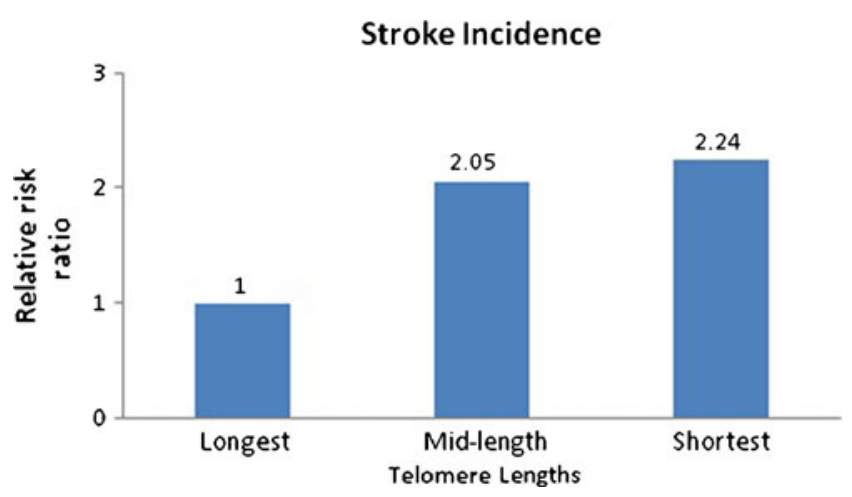

Fig. 2 Relation of telomere length to incidence of stroke (Data adapted from Willeit et al. [20])

\section{Cancer}

Cancer has long been known to be associated with telomere function (see textbook review) [8], although the relationship has proven increasingly complex. On the one hand, most cancers develop the ability to maintain telomere lengths to "bypass" the enforced cell senescence occasioned by short telomeres, which would otherwise limit tumor growth. On the other hand, long telomeres have the effect of maintaining genomic stability and both preventing the initial gene damage that underlies many malignancies and (through enhanced genomic repair) causing some early genomic damage to be repaired, thereby preventing such cells from progressing to clinical malignancy. Whatever the mechanisms, which are no doubt more complex than we yet realize, cancer cells are often characterized by short (but maintained) telomere lengths.

Therefore, short telomere length has long been posited as a useful biomarker in both the diagnosis and prognosis of many clinical cancers and the data have borne this out (Figs. 3 and 4) [30]. Whether or not telomere lengths also can be used to predict the likelihood of cancer is more questionable [22]. If telomere shortening is a secondary (rather than primary) phenomenon, occurring as a result of malignant transformation and largely after the cancer is diagnosed, then it may offer little or no value as a predictive biomarker [31]. Whether a direct result of malignant transformation or due to the dysplasia occurring in a "preneoplastic field of chronic inflammation" [32], telomere shortening does occur quite early in the malignant process [33] and may well offer potential as an initial diagnostic biomarker.

Telomere length is questionable as a biomarker for predicting the likelihood of getting cancer, but it is a useful biomarker in the staging and prognosis of cancers, once diagnosed.

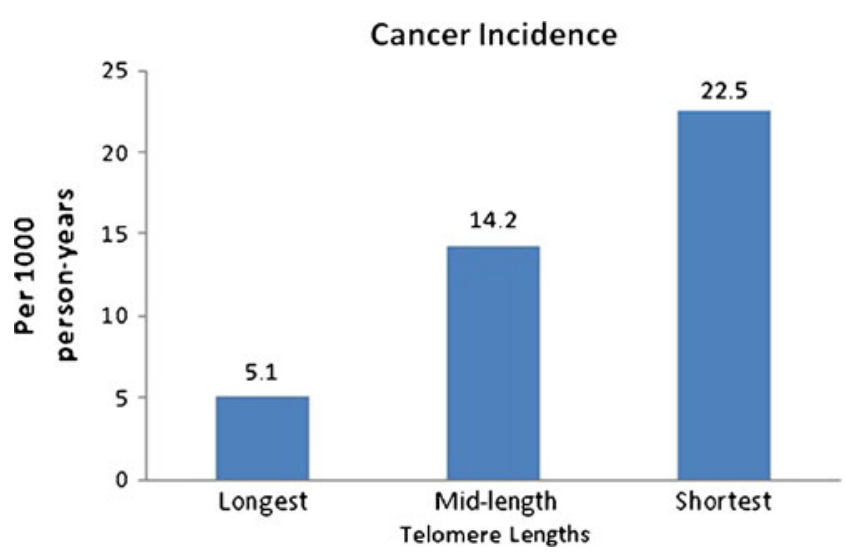

Fig. 3 Relation of telomere length to incidence of cancer (Data adapted from Willeit et al. [30]) 


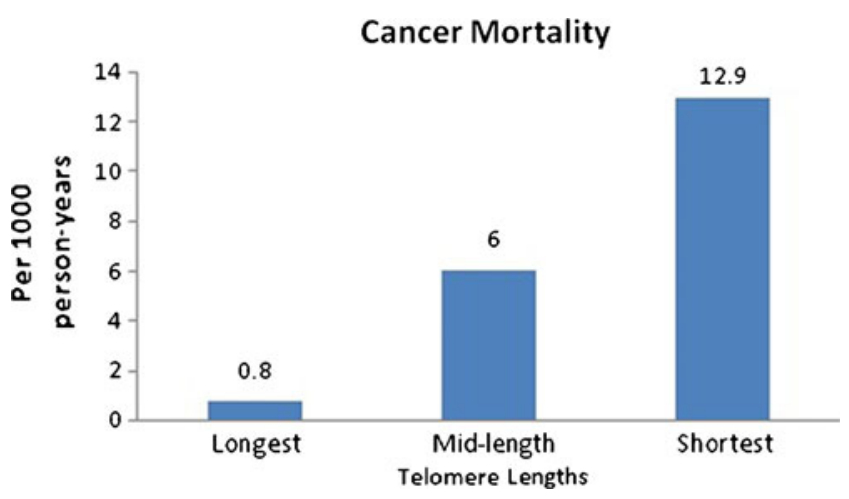

Fig. 4 Relation of telomere length to cancer mortality (Data adapted from Willeit et al. [30])

\section{Other Diseases}

Telomere length has been evaluated as a biomarker for a number of other diseases. The broadest category of such diseases is those related to infection, inflammation, anemias, and/or autoimmune disease [34]. This potpourri of diseases share a common overlapping function in maintaining immunity, albeit poorly so in the case of clinical disease. As in the case of cardiovascular disease, the relationship with diagnosis or disease events is well-supported, but the relationship with mortality is much less credible [19]. Telomere length is associated with inflammation [35], particularly in chronic viral infections [12], chronic obstructive pulmonary disease (COPD) [36], fibrotic disease [37], pulmonary fibrosis [38], tobacco use [39], and other immune-related diseases [37]. There is extensive literature on the role of telomere lengths in aplastic anemia, but while telomere lengths show no predictive value for the initial therapeutic response, they do offer predictive value for relapse, clonal evolution, and survival [40].

Telomere length also has been looked at as a potential predictor of central nervous system aging, but results have been disappointing [41, 42]. The possibility of using telomere lengths in assessing Alzheimer's disease is more tantalizing, but has several inherent obstacles. The first of these, that neurons don't divide and hence show no telomere shortening, is a straw man, because the disease is almost certain due to the activation of microglia, cells that clearly do show progressive telomere shortening [43] and that may well underlie and time the progression of Alzheimer's disease. The second obstacle, however, is that the assessment of microglial telomere lengths requires brain biopsy, rendering it impractical. The extent to which circulating leukocytes might serve as indicators of microglial function, and more importantly microglial telomere lengths, is unknown.

Finally, at least one group has suggested that lens transparency might be directly related to leukocyte telomere lengths [44], although there is little current data to substantiate the telomere length as a biomarker that may predict future lens function.

\section{Interventions in Telomere Length}

The optimal biomarker permits intervention. While chronologic age, for example, may be informative, we cannot intervene in the chronologic age per se. Equally, in the case of familial history, which is a significant biomarker for many diseases, we cannot intervene and change the family history per se. In the case of the actual underlying genetics, however, there is an obvious potential to alter the genetic biomarkers; we anticipate our ability to alter the alleles that result in certain diseases, current technical challenges and disappointing clinical setbacks notwithstanding. Many current biomarkers (such as serum cholesterol, blood pressure, and smoking status) are reasonably susceptible to medical intervention, but while the clinical response is heartening, it is far from perfect, whether in terms of outcome, side effects, cost, recurrence of the disease process, or patient mortality. Current literature supports the potential of telomere length as a biomarker for many diseases, but is there any potential for intervention? Is it, like age itself, useful but not alterable? Or is it, like serum cholesterol, a biomarker that we can change and, with it, change the disease process and clinical outcome? Over the past decade $[8,26]$, there has been a growing interest in the potential benefits of maintaining or lengthening telomeres as a clinical intervention in human patients [34].

A number of interventions have been suggested as being able to extend (or maintain) telomere lengths in vivo, including several steroids such as sex steroids [37] and several astralagoside compounds [45 $]$, and other potential agents [39] or even behavioral changes. Telomere lengths have been shown to be alterable in vitro for more than a decade and in vivo, in mice, as well [45•], resulting in improvement in agerelated pathology [46]. An informal human clinical trial using a telomerase activator has been underway for several years, and the initial results suggest that we can intervene in telomere lengths, with good clinical results [47••].

To date, the single most effective compound (and the only compound used in human studies) has been a small molecule activator of telomerase, derived from the root of Astragalus membranaceus, a group of steroids collectively termed astagalosides and often designated TA-65 in the literature that increases the mean telomere length and decreases the percentage of critically short telomeres both in vitro and in vivo both in mice and in humans. The mouse studies show improvements in glucose tolerance, osteoporosis, skin function, and other age-related diseases without any increase in the incidence of cancer [45•]. The initial 
human study demonstrated clear improvements in immune function, again without any demonstrated increase in cancer or other side effects $[47 \bullet \bullet]$.

Telomere lengths not only are a reasonable biomarker for a number of age-related diseases, but also have the advantage of being amenable to clinical intervention. To date, there is at least one clinically available compound that has been demonstrated to increase telomere lengths in both animals and humans.

\section{Conclusions}

Telomere lengths, usually measured in circulating leukocytes, are an effective biomarker for a number of agerelated diseases, offering both diagnostic and prognostic information in many cases, although their utility for predictive mortality is questionable. Telomere lengths are better than chronologic age in assessing the overall aging process in human patients. In the case of clinical disease, they are reasonably effective in assessing cardiovascular disease status and for diseases with an inflammatory or immune component. Nonetheless, they need to be used with some care; current techniques need to be improved and clinicians need to beware of the reliability (especially between techniques, between labs, and even within labs) when using telomere lengths to assess age-related disease. Finally, the literature is relatively new and somewhat scant, and the techniques of measuring leukocyte telomere lengths will require greater depth and broader clinical use before it can provide clear improvement over the more common biomarkers on which most clinical literature is based.

The potential for telomere lengths as biomarkers and the potential for telomerase activators as a clinical intervention are promising and are generally supported by current data.

Disclosure No potential conflicts of interest relevant to this article were reported.

\section{References}

Papers of particular interest, published recently, have been highlighted as:

- Of importance

•- Of major importance

1. Fossel M. Human aging and progeria. J Ped Endo Metab. 2000;13 (6):1477-81.

2. Fossel M. Reversing human aging. New York, New York: William Morrow \& Co.; 1996.

3. Banks D, Fossel M. Telomeres, cancer, and aging: altering the human lifespan. JAMA. 1997;278:1345-8.

4. Fossel M. Telomeres and the aging cell: implications for human health? JAMA. 1998;279:1732-5.
5. Fossel M. Cell senescence in human aging: a review of the theory. In Vivo. 2000;14:29-34.

6. Wong LSM, Huzen J, de Boer RA, van Gilst WH, van Veldhuisen DJ, van der Harst P. Telomere length of circulating leukocyte subpopulations and buccal cells in patients with ischemic heart failure and their offspring. PLoS One. 2011;6:e23118.

7. Svenson U, Nordfjall K, Baird D, Roger L, Osterman P, Hellenius ML, Roos G. Blood cell telomere length is a dynamic feature. PLoS One. 2011;6:e21485.

8. Fossel M. Cells, Aging, and human disease. Oxford University Press [academic text], 2004.

9. - Trudeau MA, Wong JMY. Genetic variations in telomere maintenance, with implications on tissue renewal capacity and chronic disease pathologies. Curr Pharmacogenomics Person Med. 2010;8:7-24. This is a clear, broad summary of the value of telomere biology for age-related human disease, particularly those that not only involve the expected, gradual loss of telomere length over the course of the human lifespan, but also those diseases that involve faulty telomere maintenance. They review several known abnormalities of telomerase maintenance and the implications for human disease, particularly chronic inflammatory disease, as well as the implications for translational research and intervention.

10. Leslie M. Are telomere tests ready for prime time? Science. 2011;332:414-5.

11. Van de Berg PJEJ, Griffiths SJ, Yong SL, Macaulay R, Bemelman FJ, Jackson S, Henson SM, ten Berge IJM, Akbar AN, van Lier RAW. Cytomegalomvirus infection reduces telomere length of the circulating T cell pool. J Immunol. 2010;184:3417-23.

12. Hoare M, Gelson WTH, Das A, Fletcher JM, Davies S, Curran MD, Vowler SL, Maini MK, Akbar AN, Alexander GJM. CD4+ Tlymphocyte telomere length is related to fibrosis stage, clinical outcome and treatment response in chronic hepatitis $\mathrm{C}$ virus infection. J Hepatol. 2010;53:252-60.

13. Effros RB, Allsopp R, Chiu CP, Hausner MA, Hirji K, Wang L, Harley CB, Villeponteau B, West M. Shortened telomere in the expanded CD28-CD8+ cell subset in HIV disease implicate replicative senescence in HIV pathogenesis. AIDS. 1996;10: F17-22.

14. Aviv A, Hunt SC, Lin J, Cao X, Kimura M, Blackburn E. Impartial comparative analysis of measurement of leukocyte telomere length/DNA content by Southern blots and qPC. Nucleic Acids Res. 2011;39:134.

15. Kimura M, Aviv A. Measurement of telomere DNA content by dot blot analysis. Nucl Acids Res. 2011;39:e84.

16. Mather KA, Jorm AF, Parslow RA, Christensen H. Is telomere length a biomarker of aging? A review. J Gerontol A Biol Sci Med Sci. 2011;66A:202-13.

17. Calado RT, Regal JA, Kleiner DE, Schrump DS, Peterson NR, Pons V, Chanock SJ, Lansdorp PM, Young NS. A Spectrum of Severe Familial Liver Disorders Associate with Telomerase Mutations. PLoS One. 2009;4:e7926.

18. Sanders JL, Fitzpatrick AL, Boudreau RM, Arnold AM, Aviv A, Kimura M, Fried LF, Harris TB, Newman AB. Leukocyte telomere length is associated with noninvasively measured age-related disease: the cardiovascular health study. J Gerontol A Biol Sci Med Sci. 2011 Sep 20 [Epub ahead of print].

19. Fitzpatrick AL, Kronmal RA, Kimura M, Gardner JP, Psaty BM, Jenny NS, Tracy RP, Hardikar S, Aviv A. Leukocyte telomere length and mortality in the cardiovascular health study. J Gerontol A Biol Sci Med Sci. 2011;66:421-9.

20. Willeit P, Willeit J, Brandstatter A, Ehrlenbach S, Mayr A, Gasperi A, Weger S, Oberhollenzer F, Reindl M, Kronenberg F, Kiechl A. Cellular aging reflected by leukocyte telomere length predicts advanced atherosclerosis and cardiovascular disease risk. Arterioscler Thromb Vasc Biol. 2010;30:1649-56. 
21. Hoffmann J, Spyridopoulos I. Telomere length in cardiovascular disease: new challenges in measuring this marker of cardiovascular aging. Futur Cardiol. 2011;7:789-803.

22. Houben JMJ, Giltay EJ, Rius-Ottenheim N, Hageman GJ, Kromhout D. Telomere length and mortality in elderly men: the zutphen elderly study. J Gerontol A Biol Sci Med Sci. 2011;66A:38-44.

23. Strandberg TE, Saijonmaa O, Tilvis RS, Pitkala KH, Strandberg AY, Miettinen TA, Fyhrquist F. Association of telomere length in older men with mortality and midlife body mass index and smoking. J Gerontol A Biol Sci Med Sci. 2011;66A:815-20.

24. Weischer M, Bojesen SE, Cawthon RM, Freiberg JJ, TybjaergHansen A, Nordestgaard BG. Short telomere length, myocardial infarction, ischemic heart disease, and early death. Arteriosclerosis, Thrombosis, and Vascular Biology, 2011; 1111.237271.

25. Huzen J, Peeters W, de Boer RA, Moll FL, Wong LSM, Codd V, de Kleijn DPV, de Smet BJGL, van Veldhuisen DJ, Samani NJ, van Gilst WH, Pasterkamp G, van der Harst P. Circulating leukocyte and carotid atherosclerotic plaque telomere length interrelation, association with plaque characteristics, and restenosis after endarterectomy. Arterioscler Thromb Vasc Biol. 2011;31:1219-25.

26. Serrano AL, Andrés V. Telomeres and cardiovascular disease: does size matter? Circ Res. 2004;19(94):575-84.

27. Ozsarlak-Sozer G, Kerry Z, Gokce G, Oran I, Topcu Z. Oxidative stress in relation to telomere length maintenance in vascular smooth muscle cells following balloon angioplasty. J Physiol Biochem. 2011;67:35-42.

28. Fyhrquist F, Silventoinen K, Saijonmaa O, Kontula K, Devereux RB, de Faire U, Os I, Dahlöf B. Telomere length and cardiovascular risk in hypertensive patients with left ventricular hypertrophy: the LIFE study. J Hum Hypertens. 2011;25:711-8.

29. Calvert PA, Liew TV, Gorenne I, Clarke M, Costopoulus C, Obaid DR, O'Sullivan M, Shapiro LM, McNab DC, Densem CG, Schofield PM, Braganza D, Clarke SC, Ray KK, West NEJ, Bennett MR. Leukocyte telomere length is associated with high-risk plaques on virtual histology intravascular ultrasound and increased proinflammatory activity. Arterioscler Thromb Vasc Biol. 2001;31:2157-64.

30. Willeit P, Willeit J, Mayr A, Weger S, Oberhollenzer F, Brandstatter A, Kronenberg F, Kiechl S. Telomere length and risk of incident cancer and cancer mortality. JAMA. 2010;304:69-75.

31. Pooley KA, Sandhu MS, Tyrer J, Shah M, Driver KE, Luben RN, Bingham SA, Ponder BAJ, Pharoah PDP, Khaw KT, Easton DF, Dunning AM. Telomere length in prospective and retrospective cancer case-control studies. Cancer Res. 2010;70:3170-5.

32. Risques RA, Lai LA, Himmetoglu C, Ebaee A, Li L, Feng Z, Bronner MP, Al-Lahham B, Kowdley KV, Lindor KD, Rabinovitch PS, Brentnall TA. Ulcerative colitis-associated colorectal cancer arises in a field of short telomeres, senescence, and inflammation. Cancer Res. 2011;71:1669-79.

33. Rampazzo E, Bertorelle R, Serra L, Terrin L, Candiotto C, Pucciarelli S, Del Bianco P, Nitti D, De Rossi A. Relationship between telomere shortening, genetic instability, and site of tumour origin in colorectal cancers. Br J Cancer. 2010;102:1300-5.

34. Andrews NP, Fujii H, Goronzy JJ, Weyand CM. Telomeres and immunological diseases of aging. Gerontology. 2010;56:390-403.

35. O'Donovan A, Pantell MS, Puterman E, Dhabhar FS, Blackburn EH, Yaffe K, Cawthon RM, Opresko PL, Hsueh WC, Satterfield S, Newman AB, Ayonayon HN, Rubin SM, Harris TB, Epel ES.
Cumulative inflammatory load is associated with short leukocyte telomere length in the health, aging and body composition study. PLoS One. 2011;6:e19687.

36. Savale L, Chaouat A, Bastuji-Garin S, Marcos E, Boyer L, Maitre B, Sarni M, Housset B, Weitzenblum E, Matrat M, Le Corvoisier P, Rideau D, Boczkowski J, Dubois-Rande JL, Chouaid C, Adnot S. Shortened telomeres in circulating leukocytes of patients with chronic obstructive pulmonary disease. Am J Respir Crit Care Med. 2009;179:566-71.

37. Calado RT, Young NS. Telomere diseases. NEJM. 2009;361:2353-65.

38. Cronkhite JT, Xing C, Raghu G, Chin KM, Torres F, Rosenblatt RL, Garcia CK. Telomere shortening in familial and sporadic pulmonary fibrosis. Am J Respir Crit Care Med. 2008;178:729-37.

39. Babizhayev MA, Savel'yeva EL, Moskvina SN, Yegorov YE. Biologic age, and an independent predictor of survival and therapeutic treatment requirement associated with smoking behavior. Am J Ther. 2011;18:e209-26.

40. Scheinberg P, Cooper JN, Sloand EM, Wu CO, Colado RT, Young NS. Association of telomere length of peripheral blood leukocytes with hematopoietic relapse, malignant transformation, and survival in severe aplastic anemia. JAMA. 2010;304:1358-64.

41. Devore EE, Prescott J, De Vivo I, Grodstein F. Relative telomere length and cognitive decline in the nurses' health. Study. Neurosci Lett. 2011;492:15-8.

42. Insel KC, Merkle CJ, Hsiao CP, Vidrine AN, Montgomery DW. Biomarkers for cognitive aging-Part I: Telomere length, blood pressure and cognition among individuals with hypertension. Biol Res Nurs 2011; 1099800411406433.

43. Flanary BF, Streit WJ. Progressive telomere shortening occurs in cultured rat microglia, but not astrocytes. Glia. 2003;45:75-88.

44. Sanders JL, Iannaccone A, Boudreau RM, Conley YP, Opesko PL, Hsueh WC, Cummings SR, Cawthon RM, Harris TB, Nalls MA, Kritchevsky SB, Newman AB. The association of cataract with leukocyte telomere length in older adults: defining a new marker of aging. J Gerontol A Biol Sci Med Sci. 2011;66A:639-45.

45. De Jesus BB, Schneeberger K, Vera E, Tejera A, Harley CB, Blasco MA. The telomerase activator TA-65 elongates short telomeres and increases health span of adult old mice without increasing cancer incidence. Aging Cell. 2011;10:604-21. This key research group summarizes their recent work, in which they used a telomerase activator (TA-65, an astragaloside) to elongate short telomeres in mice. Treated mice have less DNA damage than untreated mice, as well as showing increased glucose tolerance, decreased osteoporosis, and improved "skin fitness," without any increase in their incidence of cancer.

46. Donate LE, Blasco MA. Telomeres in cancer and ageing. Phil Trans R Soc B. 2011;366:76-84.

47.• Harley CB Liu W, Blasco M, Vera E, Andrews WH, Briggs LA, Raffaele JM. A natural product telomerase activator as part of a health maintenance program. Rejuvenation Research. 2011;14:4556. This is the first reported study of telomere extension as a human clinical intervention, reporting the first year of data from an uncontrolled human trial, begun in 2007, focusing on the immune system. At 6-12 months, the telomerase activator (TA-65, an astragaloside) was shown to lengthen critically short leukocyte telomeres and change the proportion of circulating leukocytes to a more "youthful" profile. 